\title{
PRODUCTIVIDAD Y TASA DE PLUSVALOR A NIVEL INTERNACIONAL: EVALUACIÓN EMPÍRICA ${ }^{1}$
}

EXPOSITOR

Mario César Sánchez

INVESTIGADORES

Mario César Sánchez

Gloria Martínez González

Alejandro Valle

Departamento de Economía
El presente trabajo examina la relación entre productividad y tasa de plusvalor, y si en los países desarrollados (PD) -con mayor productividad-, se verifica una tasa de plusvalor mayor a la correspondiente a los países subdesarrollados (PsD) de acuerdo con la conjetura teórica marxista de dicha relación a nivel internacional. Para ello, se emplearon tablas de contingencia, análisis multivariado mediante clústeres y análisis econométrico de datos de panel de efectos fijos. En este último se usaron diversas bases de datos, cuyos periodos de estudio van desde la posguerra a la primera década del siglo XXI. La investigación encuentra que dentro de cada bloque de países, desarrollados y subdesarrollados, se encuentra una relación directa entre $\pi$ y TP, que al incrementarse $10 \%$ la productividad en los PD, su TP crece en $1.7 \%$ y que en el caso de los países subdesarrollados este incremento es casi del 3\%.

\section{ANTECEDENTES.}

Aunque este problema de niveles de tasas de plusvalor mayores en PD se conoce, no ha recibido explicación por parte de los especialistas. Además, debe de decirse que no se trata sólo de una paradoja de la teoría marxista. La economía neoclásica en sus funciones de producción obtiene participaciones del trabajo en un orden del $60 \%$ para los salarios y $40 \%$ para el capital en PD, para los PsD las participaciones son inversas, el porqué de esta regularidad no ha sido en modo alguno explicado por el mainstream neoclásico. Algunas de las economistas que han estudiado este problema de tasas de plusvalor diferenciales y desarrollo son Martínez (2003) y Martínez y Valle (2011). Este trabajo se basa en las contribuciones de esta autora y de este autor. Martínez fue la primera en documentar la paradoja de tasas de plusvalor mayores en PsD, si bien Amsden (1981), ya había advertido en la manufactura la diferencial tasa de plusvalor entre países de 
diferente desarrollo, mientras que otros autores como Kalmans (1993) analizaron las diferenciales entre países desarrollados, pero no entre PD versus PsD.

A continuación se muestran las composiciones de capital, productividad y tasa de plusvalía para una amplia muestra mundial de 1995 a 2008, ver Gráficas 1, 2 y 3. Los datos son obtenidos del Extended Penn World Table v.4., y la tasa de plusvalía se obtiene gruesamente como la relación de las ganancias entre los salarios para los países en que se disponían estos datos en la base señalada.
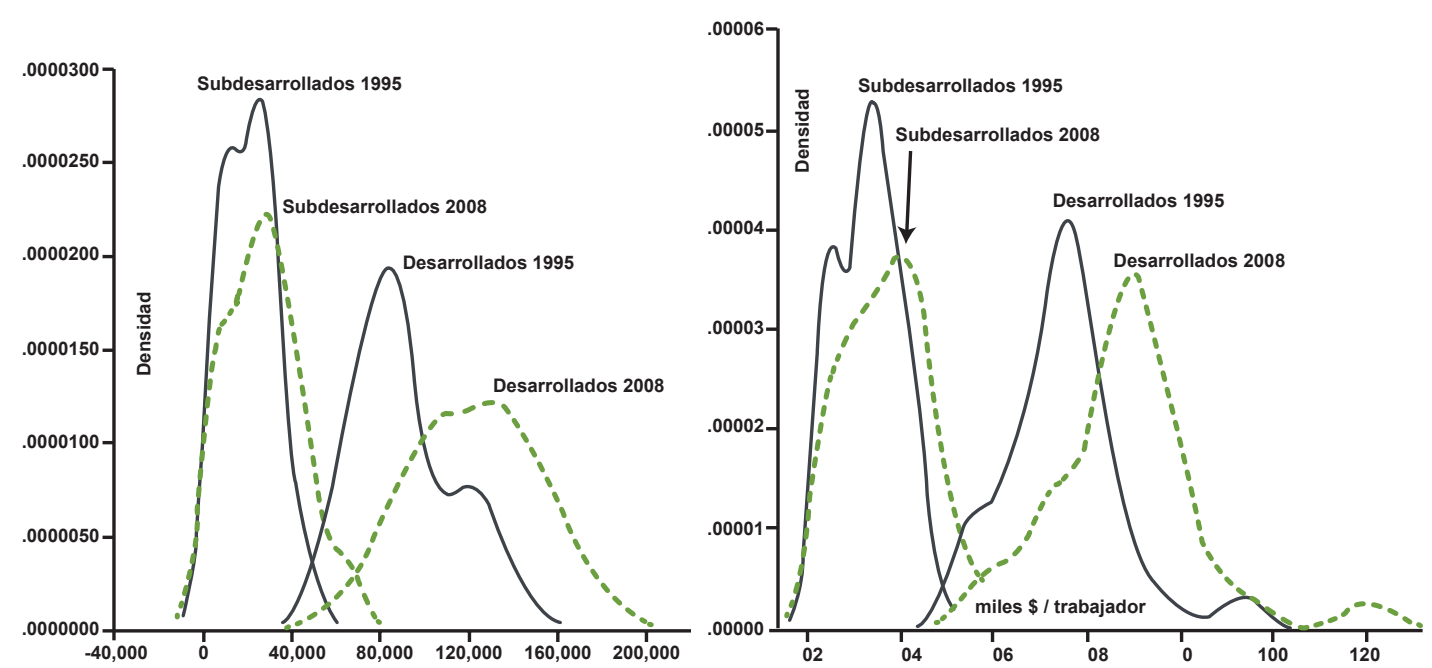

Gráfica 1 Y 2. Capital/Trabajo y Productividad, 72 Paises SEleccionados, 1995-2008. Conjunto de Países Desarrollados (28) y SubdeSARRollados (44)

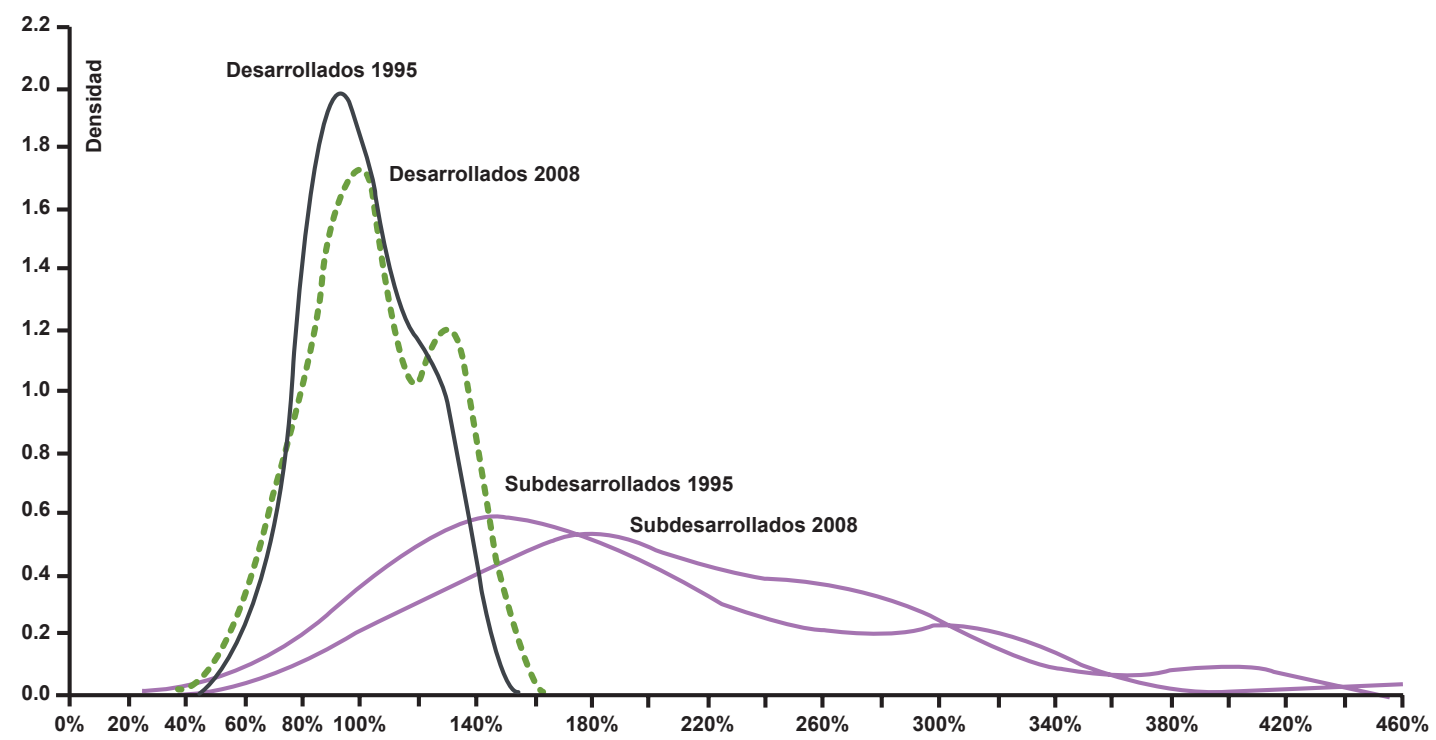

Gráfica 3. Tasa de Plusvalor. Ganancias/Salarios. 72 Paises Seleccionados, 1995-2008.

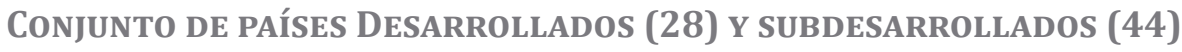

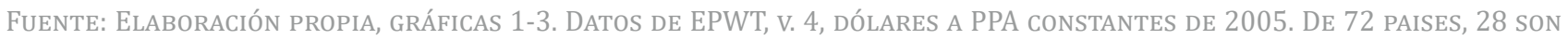
DESARROLLADOS, DE ACUERDO A SU PRODUCTIVIDAD RELATIVA A LA MEDIA MUNDIAL. 
Las funciones de densidad nos permiten ver los valores principales de las distribuciones de cada variable, en los ejes horizontales están las unidades de éstas (ver Gráficas 1, 2 y 3). De este modo, y de acuerdo a las modas de las funciones, el capital por trabajador de los PsD ronda los $30 \mathrm{mmd}$ por trabajador y su avance en 2008 fue ínfimo. En contraste, los PD de $80 \mathrm{mmd}$ en 1995 pasaron a más de 140 mmd en 2008, es decir, la composición pasó a ser en 2008 de ¡casi 5 veces más que la de los PsD! Como resultado de lo anterior, en términos de productividad laboral, los PD en 2008 tenían una productividad promedio de casi $80 \mathrm{mmd}$ por trabajador, los PsD sólo alcanzaron los $20 \mathrm{mmd}$; la relación es pues, de casi 4 a 1 . Pero ¿qué sucedió con la tasa de plusvalía en 2008?, ciertamente creció para los dos conjuntos de países, teniendo un promedio de $180 \%$ para los PsD y $100 \%$ para los PD, es decir que, contrario a los esperado, los niveles de tasa de plusvalor fueron de 1.8 a 1 "a favor" de los países subdesarrollados.

El problema y la paradoja quedan planteados: ¿Significa esto que la relación positiva planteada por Marx entre productividad y tasa de plusvalor no es cierta?, no es así, como se desarrollará más adelante dentro del conjunto de cada tipo de país, la aseveración de Marx es correcta.

Un análisis simple de la relación de productividad y tasas de plusvalía parecería plantear una relación falsamente inversa, eso es lo que se ve en el Gráfico 4, en la línea negra descendente.

Puede ahora plantearse el modelo de efectos fijos: $\boldsymbol{l n}\left(\boldsymbol{p}^{\prime}\right)_{i t}=\boldsymbol{\beta}_{\boldsymbol{1}} \boldsymbol{\operatorname { l n }}(\boldsymbol{\pi})$ ${ }_{i t}+\alpha_{i}+\boldsymbol{u}_{i t}$, donde: $\mathrm{p}^{\prime}=$ ganancias / salarios es una variable proxy de la tasa de plusvalía, $\boldsymbol{\pi}=\mathrm{PIB} /$ Empleo que expresa la productividad, i país y t el tiempo. De este modo, se tienen $\mathrm{N}=\mathrm{i}$ $\times \mathrm{t}=72 \times 14=1,008$ observaciones. Analizan la relación entre tasa de plusvalor y productividad tanto para el conjunto de países como para los subconjuntos de países desarrollados y subdesarrollados por separado, que son definidos según su productividad superior o inferior a la productividad media mundial, respectivamente. Los resultados se muestran en el Cuadro 1.
Conjunto de países:

(Ecuación 1)

Países desarrollados:

(Ecuación 2)

Países subdesarrollados:

(Ecuación 3) $\ln \left(p^{\prime}\right)_{\mathrm{it}=} 0.2567 \ln (\pi) i t-2.1674+$ uit,

t-estad. (20.7150) (-17.5014)

$\ln \left(p^{\prime}\right)_{\mathrm{it}}=0.1772 \ln (\pi) i t-1.9417+$ uit, t-estad. (8.4134) (-8.3983)

$\ln \left(p^{\prime}\right) i t=0.2742 \ln (\pi) i t-1.9184+u i t$, t-estad. (19.592) (-14.5361) 


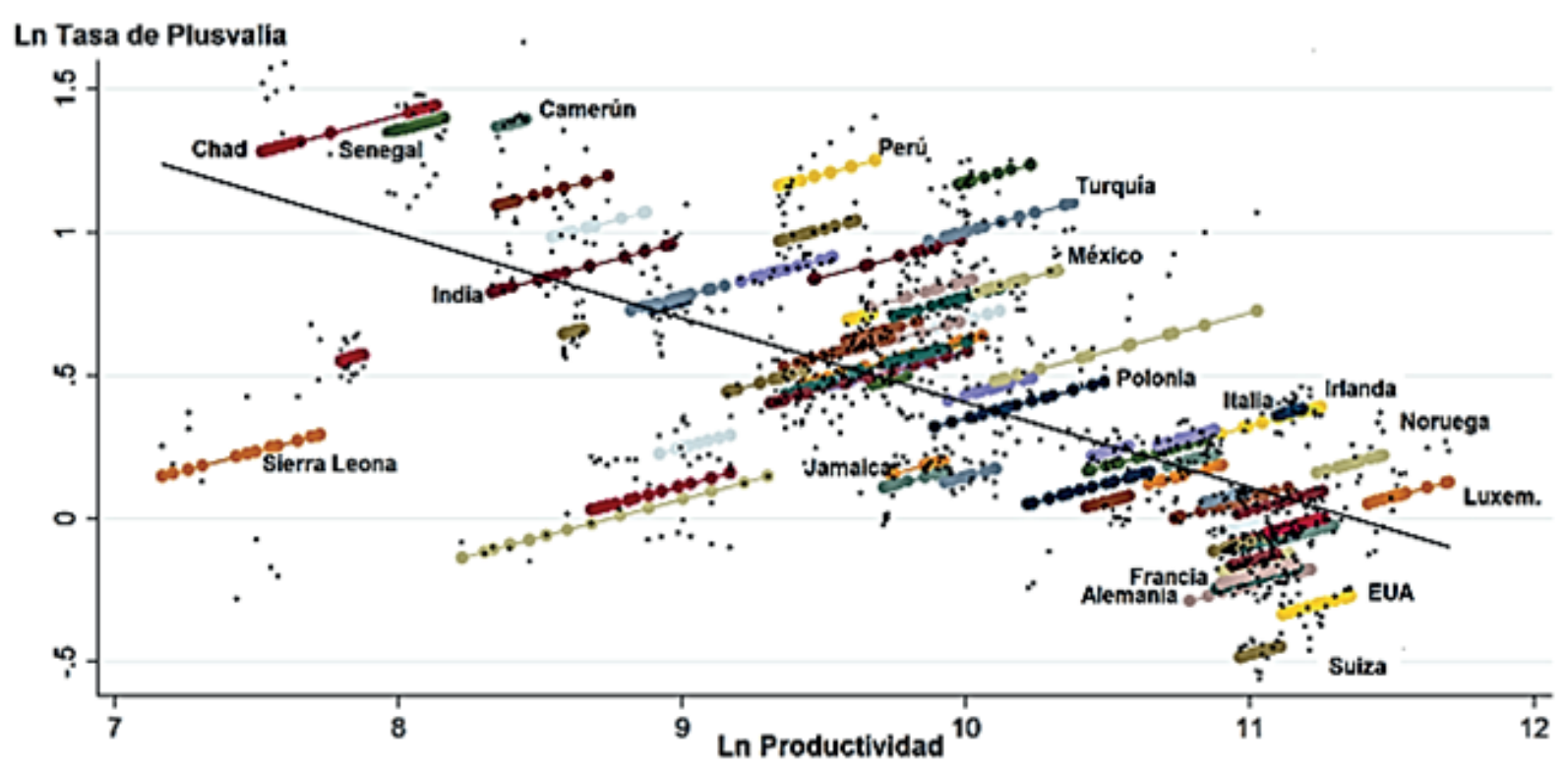

GRÁFICA 4. LA RELACIÓN DIRECTA POR PAÍSES DE LA TASA DE PLUSVALÍA POR LA PRODUCTIVIDAD. EFECTOS FIJOS CON MCG. 72 PAISES SELECCIONADOS, 1995-2008.

FUENTE: ELABORACIÓN PROPIA CON DATOS DE EPWT, V. 4, LA LÍNEA NEGRA ESTIMADA POR MCO SIMPLE APARENTA UNA RELACIÓN INVERSA. LOS PUNTOS NEGROS SON LAS COORDENADAS $\left(\pi_{i}, \mathrm{P}^{\prime}{ }^{\prime}\right)$, EN CAMBIO, LOS PUNTOS CONECTADOS SON LAS ESTIMACIONES MEDIANTE EFECTOS FIJOS QUE MUESTRAN UNA RELACIÓN DIRECTA EN LA GENERALIDAD DE CASOS.

La prueba de Hausman define que los modelos deben estimarse bajo efectos fijos y no aleatorios. No existe raíz unitaria en ellos, según la prueba de Levin-Li-Chu. Además, son paneles donde $i>t$, de modo que el problema más relevante es la heteroscedasticidad, por lo que se procedió a obtener mínimos cuadrados generalizados, ponderando por sección cruzada y con errores consistentes de White. Además, dado que los modelos son log-log, los estimadores obtenidos son elasticidades. En los anteriores se encontró que los países desarrollados presentan un menor efecto de la productividad sobre la tasa de plusvalor, $54 \%$ menos elasticidad que los países subdesarrollados, ya que se observa que $\beta_{11}=0.177<\beta_{12}=0.274$.

\section{CONCLUSIONES.}

Hay que destacar algunos puntos de lo desarrollado hasta ahora.

1) Existe una evidencia indiscutible de que los PsD tienen un nivel de tasa de plusvalor mayor a los PD, siempre con las cuentas nacionales actuales y, por tanto, bajo los precios de mercado vigentes; 2) por esta razón es que, además, al ser analizados en conjunto la relación entre tasa de productividad y tasa de plusvalor parece inversa; 3 ) pero existen razones para pensar que esto es mera apariencia. Un análisis más cuidadoso mediante técnicas econométricas de datos de panel y 
efectos fijos nos permite indagar que la relación entre las dos variables se cumple dentro de cada conjunto de países (Ecuación 1 y 2), como bien lo ha desarrollado Martínez (2003); 4) no sólo eso, incluso en el conjunto de países, si se hace la separación del nivel particular de cada país (efectos fijos), la relación también es directa (Ecuación 3); 5) de este modo, todo parece orillar el estudio a que las transferencias del valor internacional, y los precios corrientes, transfiguran la relación directa que Marx vaticinaba, luego se impone la necesidad de tratar de evaluar el problema en términos de tasas de plusvalor ajustadas por estas transferencias.

\section{REFERENCIAS BIBLIOGRÁFICAS.}

Amsden, A. H. (1981). An international comparison of the rate of surplus value in manufacturing industry. Cambridge Journal of Economics (5): pp. 229-249.

Kalmans, R. F. (1993). The Relative Positions ofJapanese and U.S. Workers in the Postwar Period: An inpput_output study. Ph.D. New School for Sacial Research.

Martínez González, B. G. and Valle Baeza A. (2011). Differences in surplus-value rates between developed and underdeveloped countries. An analysis based on differences in productivity and capital composition. Marxism21 (Korean review), Vol. 21, special issue in english on Surplus-value Theory, Spring.

Marx, K. (1980). El Capital, Tomo I., Vol. 1, Secc. 3ạ, cap. VII. México: Editorial Siglo XXI.

\footnotetext{
${ }^{1}$ Este artículo está fue publicado y el presente escrito intenta realizar sólo un resumen. Recuperado de http://dx.doi.org/10.22201/iiec.20078951e.2019.196.63004
} 